\title{
Chronic dissection of the right aortic sinus with detachment of the aortic annulus and coronary artery
}

\author{
Jain Bhaskara Pillai, MD, ${ }^{a}$ Tirone E. David, MD, ${ }^{a}$ and Annette Vegas, MD, ${ }^{\mathrm{b}}$ Toronto, Ontario, Canada
}

\section{Clinical Summary}

A 51-year-old man was referred for aortic valve surgery because of severe aortic insufficiency (AI) and an unusual aortic root pathology. He has had AI since age 18 years. He recalls having a murmur since the age of 5 years. He remains completely asymptomatic. Because of progressive dilatation of the aortic root, he was referred

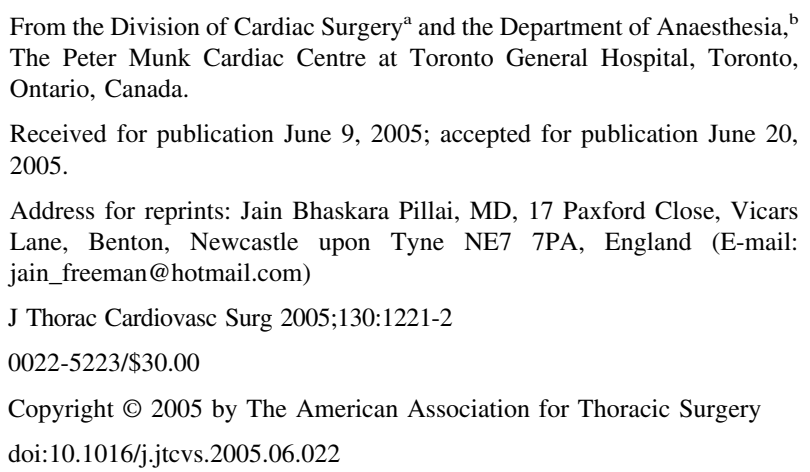

Received for publication June 9, 2005; accepted for publication June 20, 2005 .

Address for reprints: Jain Bhaskara Pillai, MD, 17 Paxford Close, Vicars Lane, Benton, Newcastle upon Tyne NE7 7PA, England (E-mail: jain_freeman@hotmail.com)

J Thorac Cardiovasc Surg 2005;130:1221-2

$0022-5223 / \$ 30.00$

Copyright (C) 2005 by The American Association for Thoracic Surgery doi:10.1016/j.jtcvs.2005.06.022

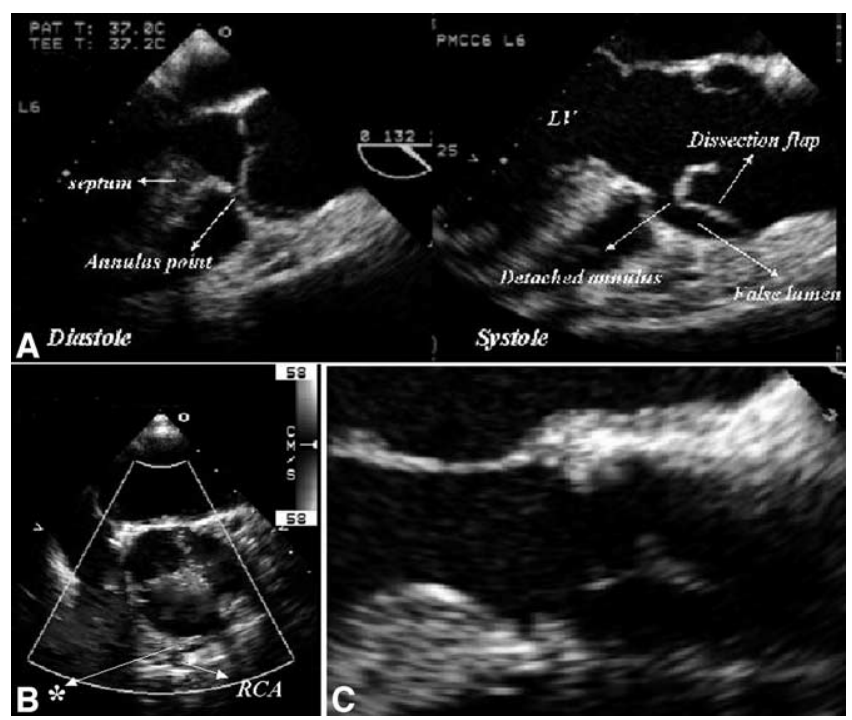

Figure 1. Transesophageal echocardiogram. A, Long-axis view before cardiopulmonary bypass, showing displacement of the right cusp and annulus into the aortic sinus. B, Short-axis view with color flow in diastole, showing central Al and flow in subannular false lumen into the right coronary artery (RCA). C, Postaortic valve-sparing procedure, showing good coaptation of the aortic cusps in diastole. for surgical intervention when it reached $50 \mathrm{~mm}$ in diameter. He is hypertensive, with no other comorbidity. Electrocardiography showed left ventricular hypertrophy. He had a normal treadmill test result.

A transesophageal echocardiogram showed a tricuspid aortic valve with severe AI. The annulus of the right cusp appeared to be detached from the aortic root and displaced into the right aortic sinus. There seemed to be a dissection involving the right aortic sinus (Figure 1, A). The false lumen appeared to arise from the left ventricular cavity and was seen to perfuse the right coronary artery (RCA; Figure 1, B). The aortic annulus was $33 \mathrm{~mm}$ in diameter, the sinuses were $50 \mathrm{~mm}$, the sinotubular junction was $40 \mathrm{~mm}$, and the proximal ascending aorta was $38 \mathrm{~mm}$.

A cardiac computed tomographic angiogram was performed on a multislice Philips Mx8000IDT 16 Scanner. The nondominant RCA appeared to be occluded at its sinus origin, and there were 3 large marginal branches fed by collaterals from the left anterior descending coronary artery (Figure 2).

At the time of the operation, the left main coronary artery was dilated at $6 \mathrm{~mm}$. The RCA orifice was occluded in the right aortic sinus, but a large volume of bright red blood appeared beneath the right aortic cusp when blood cardioplegia was injected into the left main coronary artery. The orifice of the RCA was then identified beneath the aortic valve, coming off the false lumen of the dissected right aortic sinus and membranous septum. There was a chronic dissection of the right aortic sinus that extended beyond

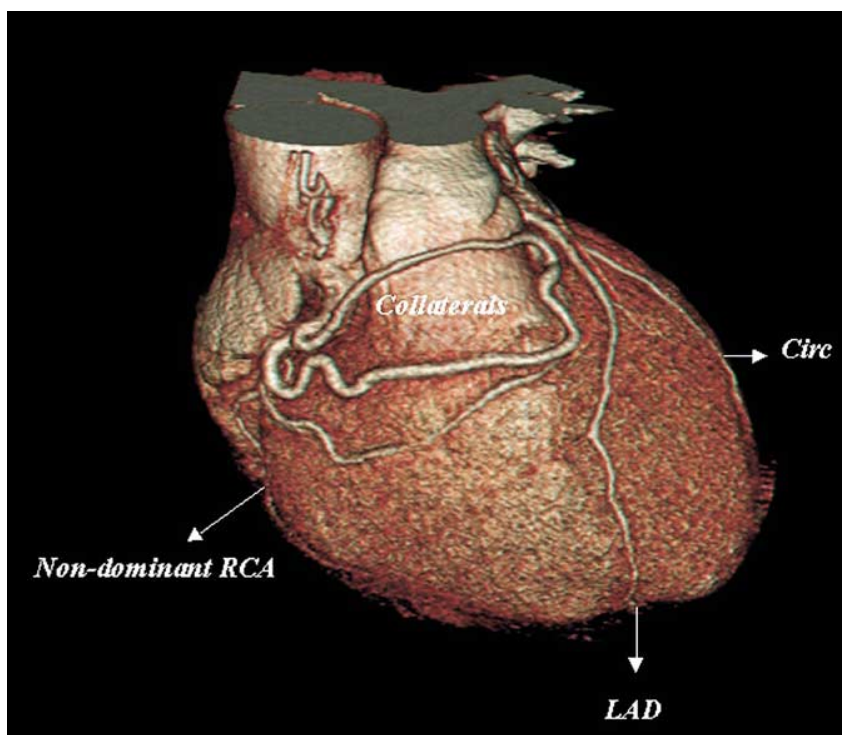

Figure 2. Computed tomographic cardiac angiogram showing the coronary anatomy. $R C A$, Right coronary artery; $L A D$, left anterior descending coronary artery. 
the aortic annulus into the aortoventricular junction and membranous septum. The RCA was surgically detached from the false lumen, along with $4 \mathrm{~mm}$ of tissue around its orifice. Cardioplegia was then administered directly into this vessel. An aortic valvesparing operation was performed with the reimplantation technique. The annulus of the right cusp and the false aneurysm wall were sutured together into the tubular Dacron graft. The left coronary artery and RCA were reimplanted. All 3 cusps required repair because of fenestrations in the commissural areas and elongation of the free margins. Postoperative echocardiography disclosed trivial AI (Figure 1,C). The patient had an uncomplicated postoperative course and was discharged 4 days later.

\section{Discussion}

Interventricular septal dissection has been described after myocardial infarction. Such a dissection chamber is isolated within the interventricular septum. ${ }^{1}$ Congenital aneurysm of the right sinus of Valsalva has been reported to rupture above the annulus, with a "wind-sock" dissection into the interventricular septum. ${ }^{2}$ Ishibashi and colleagues ${ }^{3}$ reported a chronic dissection localized to the noncoronary sinus just above the annulus.
However, we could not find any reference for a case similar to ours. The dissection might have started in the right aortic sinus at the level of the RCA and extended downward, detaching the annulus, or it is also conceivable that it commenced in the subaortic fibrous tissues and extended upward, detaching the annulus of the right aortic cusp and the RCA. The ventricular pressure feeding the RCA probably led to the development of the large collaterals to the left anterior descending coronary artery.

A localized dissection involving the aortoventricular junction and the aortic annulus is very unusual.

\section{References}

1. Hirose S, Kanemoto N, Matsuyama S. Ventricular septal dissection: a case report. J Cardiol. 1988;18:575-81.

2. Engel PJ, Held JS, van der Bel-K J, Spitz H. Echocardiographic diagnosis of congenital sinus of Valsalva aneurysm with dissection of the interventricular septum. Circulation. 1981;63:705-11.

3. Ishibashi Y, Myojin K, Ishii K, Miyazaki N, Tachibana T, Sugiki K. Chronic dissecting aneurysm of the sinus of Valsalva: report of a case [in Japanese]. Kyobu Geka. 1999;52:988-92.

\title{
aortic dissection presenting with acute ischemic stroke and undergoing thrombolytic therapy
}

Emergency surgical intervention in a patient with delayed diagnosis of

\author{
Chai-Hock Chua, MD, ${ }^{a}$ Li-Ming Lien, MD, ${ }^{\text {b }}$ Chia-Hsun Lin, MD, ${ }^{a}$ and \\ Chi-Ren Hung, MD, ${ }^{\text {a }}$ Taipei, Taiwan
}

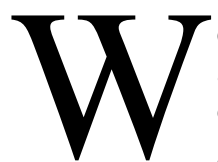

e report a patient who initially presented as having a stroke and was later discovered to have aortic dissection. Although thrombolytic therapy was given, an emergency operation was performed, and the patient was salvaged with improved but residual neurological deficit.

\footnotetext{
From the Division of Cardiovascular Surgery, Department of Surgery, ${ }^{a}$ and the Department of Neurology, ${ }^{b}$ Shin Kong Wu Ho-Su Memorial Hospital, College of Medicine, Fu Jen Catholic University, Hsinchuang, Taipei, Taiwan.

Received for publication June 2, 2005; accepted for publication June 16, 2005.

Address for reprints: Chia-Hsun Lin, MD, Shin Kong Wu Ho-Su Memorial Hospital, Cardiovascular Surgery, 95, Wen Chang Rd, shih-lin 111, Taipei 111, Taiwan E-mail: m000678@ms.skh.org.tw

J Thorac Cardiovasc Surg 2005;130:1222-4

$0022-5223 / \$ 30.00$

Copyright $\odot 2005$ by The American Association for Thoracic Surgery

doi:10.1016/j.jtcvs.2005.06.023
}

\section{Clinical Summary}

A 44-year-old man without

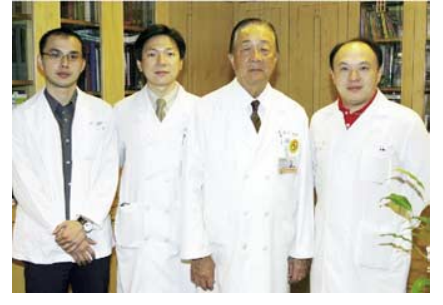

Chua, Lin, Hung, Lien (left to right) any medical history had sudden onset of left-sided weakness and slurred speech when he was drinking tea at 12:20 PM. He was sent to the emergency department at 12:40 PM. His vital signs were stable. The Glasgow Coma Scale was E3V5M6. Left central facial palsy was prominent, and left hemiplegia with reduced reflexes and positive Babinski sign were present. The National Institute of Health stroke score was 18. Head computed tomography (CT) without contrast showed no hemorrhage or hypodensity. Furthermore, transcranial color-coded duplex (TCD) scanning detected very slow flow of the right middle cerebral artery (MCA), which suggested right MCA occlusion. Therefore according to the inclusion and exclusion criteria of the thrombolytic therapy for ischemic stroke by the National Institute of Neurological Disorders and Stroke, the patient was considered eligible for intravenous recombinant tissue plasminogen activator (rt-PA). After the bolus dose of rt-PA was given at 1:30 PM for 5 minutes, the patient complained of severe left flank pain and back pain. Chest CT disclosed a Stanford type A aortic dissection extending from the ascending aorta to the common iliac arteries with involvement of the right innominate artery. Under the diag- 